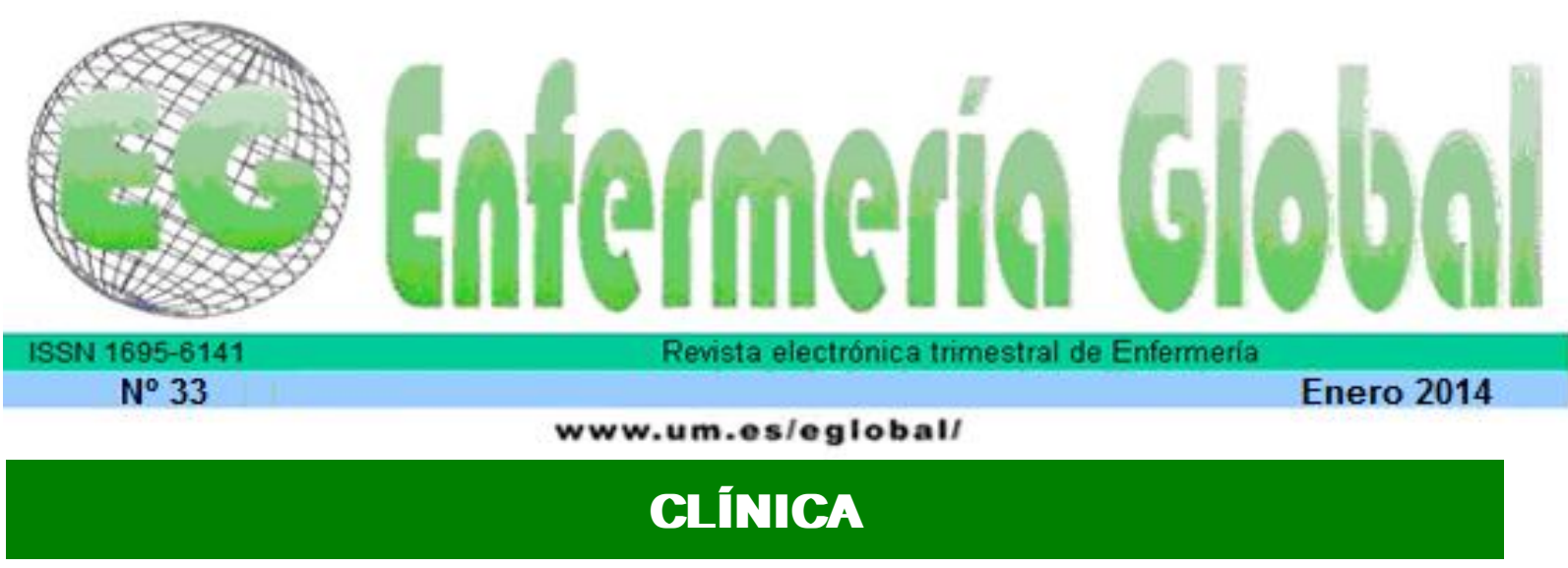

\title{
Satisfacción en la provisión de redes de apoyo social en grupos de acompañamiento a la maternidad
}

Satisfaction in providing social support networks in assistance groups for mothers

\section{*Berlanga Fernández, Sofía **Pérez Cañaveras, Rosa $M{ }^{* * *}$ Vizcaya Moreno, M Flores ****Berlanga Fernández, Francisco}

\begin{abstract}
*Área Básica de Salud (ABS) Sta. Eulalia Sur. Institut Català de la Salut. Hospitalet del Llobregat, Barcelona E-mail: sbf10@hotmail.es **Departamento de Enfermería, Facultad de Ciencias de la Salud. Universidad de Alicante.. "**Área Básica de Salud (ABS) Can Serra. Institut Català de la Salut. Hospitalet del Llobregat, Barcelona. España.
\end{abstract}

Palabras clave: Salud Materno-Infantil; Investigación Cualitativa; Atención Primaria de Salud; Atención de Enfermería; Psicología; Sociología

Keywords: Maternal and Child Health; Qualitative Research; Primary Health Care; Nursing, Psychology; Sociology

\section{RESUMEN}

Objetivos: Conocer la percepción del grado de satisfacción que tienen las madres, con hijos menores de un año y que participan en las dinámicas grupales que los CAP ofrecen, en relación al contenido y redes de provisión del Apoyo Social (AS) así como las causas que disminuyen la satisfacción en el receptor de este apoyo.

Material y métodos: Estudio con diseño cualitativo y enfoque fenomenológico. La población objeto de estudio estaba constituida por madres participantes en dinámicas grupales de cinco CAP de la provincia de Barcelona.

Como instrumento de recogida de datos se empleó la entrevista semiestructurada, entre julio de 2011 y julio de 2012; todas fueron grabadas, transcritas y analizadas.

Resultados: AS informal: la mayoría de las madres están muy satisfechas-completamente satisfechas con el AS informativo, emocional y evaluativo procedente de las enfermeras; identificándose 4 categorías que contribuyen en esta percepción: contacto profesional/accesibilidad, disparidad y/o actualización, confianza y no procede.

AS formal, la mayoría de las madres están muy satisfechas-completamente satisfechas con el AS informativo, emocional, evaluativo y técnico procedente de la pareja y madre (abuela materna); 
identificándose 8 categorías en esta percepción: disparidad y/o actualización en los consejos, exigencia, empatía, confianza, inseguridades, tiempo, distancia y no procede.

Conclusiones: Los factores identificados deberían considerarse en los planes de mejora de la satisfacción y acompañamiento de las madres en este momento de profundos cambios en su vida; dado que aportar un AS satisfactorio repercute en la promoción de la salud y prevención de la enfermedad.

\section{ABSTRACT}

Objectives: To determine the level of satisfaction of mothers of under one year-old babies who participate in group activities provided by the CAP regarding the content and Social Support (AS), support network as well as the causes that lead to a lower satisfaction level for people receiving this support.

Methods: This study includes a qualitative design and phenomenological approach. The study population consisted of mothers participating in group activities in five different CAP in the province of Barcelona. Semi-structured interviews were used as a data collection instrument between July 2011 and July 2012. They were all recorded, transcribed and analyzed.

Results: informal AS: most mothers are very or completely satisfied with the information, emotional and evaluative AS from nurses; four categories were identified, that contributed to this perception: professional contact / access, discrepancy and / or update, confidence and not applicable. Formal AS: most mothers are very or completely satisfied with the information, emotional, and technical evaluation from the couple and mother (maternal grandmother) AS; eight categories were identified in this item: discrepancy and / or update on advice, requests, empathy, trust, lack of confidence, time, distance and not appropriate.

Conclusions: The factors identified should be considered in plans to improve the satisfaction and support of mothers in this time of profound change in their life since AS provides a satisfactory impact on health promotion and disease prevention.

\section{INTRODUCCIÓN}

El embarazo, parto y postparto pueden verse como una etapa de transición, dado que se produce un recorrido entre dos periodos de tiempo estable; con sentido de flujo y movimiento; y en el que se experimentan cambios tanto en el mundo externo de la madre como en la manera en que ella los percibe, repercutiendo en su vida y haciéndola vulnerable a riesgos que afectan a su salud. Este es el motivo por el que se ha de abordar la naturaleza de las transiciones, así como las condiciones y patrones de respuesta que las madres tienen ${ }^{(1)}$.

En esta línea, Mercer nos ayuda a entender la adopción del rol maternal, término que evoluciona, al ser continuo e infinito en "convertirse en madre", y que se produce a través de diversas etapas que pueden solaparse y modificarse: compromiso y preparación; conocimiento, práctica y recuperación física; normalización e integración de la identidad maternal ${ }^{(2)}$.

Asimismo, su modelo de adopción del rol maternal está situado en círculos concéntricos de Brofenbrenner, en los que se identifican el microsistema, mesosistema y macrosistema.

El microsistema es el entorno inmediato e incluye a la familia y diversos factores como el funcionamiento familiar, las relaciones padre-madre, el apoyo social y el estrés. La adopción del rol maternal se consigue en el microsistema por medio de las interacciones padre-madre-niño. 
El mesosistema, compuesto por el cuidado diario, la escuela, el lugar de trabajo y otras entidades de la comunidad más inmediata, interactúa e influye en el microsistema; es decir, en lo que ocurre al rol maternal en el desarrollo y el niño.

Por último, el macrosistema comprende los prototipos generales dados en una cultura en particular o en la coherencia cultural que es trasmitida. De esta manera, influiría a nivel social, político y cultural sobre los otros dos sistemas ${ }^{(3)}$.

Este modelo nos orienta en la influencia y composición del entramado de redes formales e informales que proveen de apoyo social (AS) a las madres ${ }^{(3,4)}$; las cuales constituyen una de las cinco dimensiones del apoyo social; siendo las otras cuatro: la dirección del apoyo, la disposición o accesibilidad a él, la descripción o su evaluación y el contenido del mismo ${ }^{(5)}$. En relación al contenido del AS, se identifican las categorías de AS: informativo, instrumental, emocional y evaluativo ${ }^{(6)}$.

A través del AS informativo, se proporciona una orientación y guía, ayudando y acompañando al individuo en la resolución de sus problemas o situaciones. Con el $A S$ emocional las personas cuentan con relaciones en las que confían, comparten y comunican problemas o preocupaciones.

Mediante el AS instrumental o físico se ofrecerá una ayuda directa.

Y por último, con el $A S$ evaluativo, se informará a las personas sobre la manera de llevar su rol utilizando comentarios y refuerzos de conductas y actitudes ${ }^{(7,8)}$.

A estas dimensiones, se le añade el grado de satisfacción que las madres perciben de los proveedores informales y formales; el cual se sustenta en la congruencia o desacuerdo entre las expectativas de la madre y la percepción de la experiencia. Cuando ésta es ofrecida por parte de la red formal, la satisfacción de la madre se considerará un indicador de calidad además de un resultado clave para medir el cuidado ${ }^{(9)}$ y proporcionar confianza y credibilidad en los profesionales ${ }^{(10)}$.

A esto se le suma que entre las competencias más relevantes de un profesional sanitario está la capacidad de comunicación en el ámbito asistencial e interprofesional, pues influye en la satisfacción de profesionales y madres, en el cumplimiento terapéutico, la prevención de conflictos, la mejora de resultados clínicos y la eficacia de los servicios sanitarios ${ }^{(11)}$.

Por lo tanto, determinar la satisfacción constituirá una área de oportunidad para mejorar la atención proporcionada ${ }^{(9)}$, la cual puede ofrecerse en los Centros de Atención Primaria de salud (CAP).de manera individual, en consulta; o bien a través de dinámicas grupales que pretenden promocionar las vinculaciones afectivas y el desarrollo del bebé a través del masaje infantil, la lactancia materna y de grupos de soporte o acompañamiento a la maternidad.

Siguiendo esta línea argumental, el presente trabajo pretende conocer la percepción del grado de satisfacción que tienen las madres, con hijos menores de un año y que participan en las dinámicas grupales que los CAP ofrecen, en relación al contenido y redes de provisión del AS, así como las causas que disminuyen la satisfacción en el receptor de este apoyo. 


\section{MATERIAL Y MÉTODOS}

Este estudio se basa en un diseño cualitativo con enfoque fenomenológico, fundamentado en la experiencia vivida de la maternidad ${ }^{(12)}$, lo cual permite tanto una aproximación a las representaciones y discursos de las mujeres como entender los significados que ellas pueden dar a sus acciones.

La población objeto de estudio estaba constituida por madres que participaban en dinámicas grupales de cinco CAP de la provincia de Barcelona.

La selección del grupo de participantes fue intencional. Todas las madres cumplieron los criterios de inclusión: a) tener un hijo menor de un año; b) participar en una dinámica grupal como masaje infantil, apoyo/acompañamiento a la maternidad y/o lactancia materna; c) no presentar problemas idiomáticos en la comunicación; y d) que los investigadores no hubieran tenido contacto profesional con ellas para evitar posibles sesgos.

La muestra se obtuvo cuando la investigadora principal o bien informante clave (enfermera referente del menor o enfermera conductora de la dinámica grupal) contactó con estos grupos.

La saturación de la información (datos cualitativos) se consiguió con un total de 21 entrevistas, pero la muestra total la conformaban 28; ya que se pretendió asegurar que no se identificaban más hallazgos.

Para llevar a cabo esta investigación se solicitó tanto la autorización del comité de ética de investigación clínica del Instituto de Investigación en Atención Primaria (IDIAP) Jordi Gol i Gurina, emitiendo éste el certificado pertinente, como el consentimiento de las informantes tras proporcionarles la información sobre los objetivos del estudio como la metodología utilizada.

El método utilizado para la recogida de datos fue la entrevista semiestructurada.

En el transcurso de la misma, la dinámica utilizada se dividía en cuatro partes: a) presentación del entrevistado, propósito del estudio, metodología utilizada, garantizando el anonimato de los datos e insistiendo que si en algún momento era deseo el poder abandonar o interrumpir la entrevista, lo podían hacer; b) datos sociodemográficos; c) datos relacionados con los proveedores, el contenido y el grado de satisfacción con el AS recogidos a modo de gradilla; y d) motivos por el que los proveedores de AS no satisfacían completamente a las entrevistadas. Esta última parte es la que se abordó de tal manera que se dejaba hablar sin interrumpir, incidiendo en cómo las madres percibieron el AS que recibían de los distintos proveedores.

Las entrevistas se realizaron entre julio del 2011 y julio del 2012; todas fueron grabadas y posteriormente transcritas. Para el análisis de los datos cuantitativos, los datos que permitían un abordaje más cuantitativo fueron analizados con la utilización del programa informático ACCESS empleándose como estadístico la proporción.

Los datos cualitativos han sido analizados sin la utilización de programas informáticos específicos. Los textos resultantes de este proceso fueron analizados 
manualmente en su estructura (Análisis de Contenido Latente) y contenido (Análisis de Contenido Manifiesto) mediante el método de análisis de "etapa a etapa" (stage-by-stage) ideado por Burnard ${ }^{(13)}$ según los siguientes pasos: a) etiquetado de los datos y creación del índice de datos; b) categorización del contenido de los datos en las categorías principales; c) formulación de una lista de categorías, códigos y subcódigos.

Los resultados hallados fueron discutidos y contrastados con profesionales que participaban en dinámicas grupales similares.

\section{RESULTADOS}

Se realizaron un total de 29 entrevistas en los centros de atención primaria donde se visitaban las participantes en el estudio. La duración de las entrevistas fue aproximadamente de 30 a 45 minutos.

Los datos que se presentaran a continuación corresponden a: a) datos sociodemográficos; $b$ ) datos relacionados con los proveedores, el contenido y el grado de satisfacción con el AS recogidos en formato de gradilla; y c) motivos por el que los proveedores de AS no satisfacían completamente a las entrevistadas.

En relación a los datos sociodemográficos, en el estudio han participado un total de 28 madres, quienes tenían una media de edad de 33,5 años. El 57,4\% tenía estudios superiores y el $42.6 \%$ secundarios. El $89.3 \%$ eran madres primerizas. Todas tenían pareja y eran de nacionalidad española. (Tabla1). 
Tabla 1: Características sociodemográficas.

\begin{tabular}{|c|c|c|c|c|c|}
\hline Entrevistada & Edad madre & Nivel de estudios & $\mathrm{N}^{\circ}$ hijos & Pareja & Nacionalidad \\
\hline E1 & 35 & Universitarios & 1 & $\mathrm{Si}$ & Española \\
\hline E2 & 38 & Universitarios & 1 & $\mathrm{Si}$ & Española \\
\hline E3 & 36 & Universitarios & 2 & $\mathrm{Si}$ & Española \\
\hline E4 & 33 & Universitarios & 1 & $\mathrm{Si}$ & Española \\
\hline E5 & 36 & Secundarios & 2 & $\mathrm{Si}$ & Española \\
\hline E6 & 37 & Universitarios & 1 & $\mathrm{Si}$ & Española \\
\hline E7 & 35 & Secundarios & 1 & $\mathrm{Si}$ & Española \\
\hline E8 & 30 & Universitarios & 1 & $\mathrm{Si}$ & Española \\
\hline E9 & 36 & Universitarios & 1 & $\mathrm{Si}$ & Española \\
\hline E10 & 31 & Universitarios & 1 & $\mathrm{Si}$ & Española \\
\hline E11 & 36 & Universitarios & 1 & $\mathrm{Si}$ & Española \\
\hline E12 & 34 & Secundarios & 1 & $\mathrm{Si}$ & Española \\
\hline E13 & 28 & Secundarios & 1 & $\mathrm{Si}$ & Española \\
\hline E14 & 31 & Universitarios & 1 & $\mathrm{Si}$ & Española \\
\hline E15 & 37 & Universitarios & 1 & $\mathrm{Si}$ & Española \\
\hline E16 & 31 & Secundarios & 1 & $\mathrm{Si}$ & Española \\
\hline E17 & 32 & Secundarios & 1 & $\mathrm{Si}$ & Española \\
\hline E18 & 31 & Universitarios & 1 & $\mathrm{Si}$ & Española \\
\hline E19 & 30 & Universitarios & 1 & $\mathrm{Si}$ & Española \\
\hline E20 & 30 & Universitarios & 1 & $\mathrm{Si}$ & Española \\
\hline E21 & 30 & Universitarios & 1 & $\mathrm{Si}$ & Española \\
\hline E22 & 32 & Secundarios & 1 & $\mathrm{Si}$ & Española \\
\hline E23 & 36 & Secundarios & 1 & $\mathrm{Si}$ & Española \\
\hline E24 & 32 & Secundarios & 1 & $\mathrm{Si}$ & Española \\
\hline E25 & 36 & Secundarios & 1 & $\mathrm{Si}$ & Española \\
\hline E26 & 37 & Secundarios & 1 & $\mathrm{Si}$ & Española \\
\hline E27 & 31 & Secundarios & 2 & $\mathrm{Si}$ & Española \\
\hline E28 & 37 & Universitarios & 1 & $\mathrm{Si}$ & Española \\
\hline & & & & & \\
\hline & & & 1 & 1 \\
\hline
\end{tabular}

A continuación se presentan los datos relacionados con los proveedores, el contenido y el grado de satisfacción con el AS ofrecido.

Respecto al AS informativo formal, casi la totalidad de las mujeres se manifiestan muy satisfechas-completamente satisfechas cuando este apoyo procede de la enfermera $(96,41 \%)$, seguida del pediatra $(85,7)$ y menos de la mitad cuando procede de la comadrona $(46,4 \%)$.

En el AS informativo informal, la mayoría de las mujeres se manifiestan muy satisfechas-completamente satisfechas cuando este apoyo procede de la pareja $(73,6 \%)$, seguido de la madre $(57,1 \%)$, en menor medida de la hermana $(46,3 \%)$ y de las amigas (42,8\%); descendiendo casi a la mitad en el caso de los amigos $(38 \%)$ y del padre $(37,2 \%)$. No procede, en la mayoría de las ocasiones del vecino (71,4\%).

(Tabla 2). 
Tabla 2: AS informativo

\begin{tabular}{|l|c|c|c|c|c|c|}
\hline INFORMATIVO & $\begin{array}{c}\text { No } \\
\text { satisfecha }\end{array}$ & $\begin{array}{c}\text { Algo } \\
\text { satisfecha }\end{array}$ & $\begin{array}{c}\text { Moderadamente } \\
\text { satisfecha }\end{array}$ & $\begin{array}{c}\text { Muy } \\
\text { satisfecha }\end{array}$ & $\begin{array}{c}\text { Completamente } \\
\text { satisfecha }\end{array}$ & $\begin{array}{c}\text { No } \\
\text { procede }\end{array}$ \\
\hline Pareja & 0 & 3,5 & 10,7 & 37,9 & 35,7 & 10,7 \\
\hline Madre & 7,1 & 7,1 & 17,8 & 25 & 32,1 & 10,7 \\
\hline Padre & 7,1 & 7,1 & 14,2 & 17,8 & 17,8 & 32,1 \\
\hline Hermana & 3,5 & 3,5 & 3,5 & 28,5 & 17,8 & 41,3 \\
\hline Hermano & 3,5 & 7,1 & 7,1 & 10,7 & 17,8 & 53,5 \\
\hline Amigos & 3,5 & 10,7 & 17,8 & 25 & 17,8 & 25 \\
\hline Vecino & 7,1 & 7,1 & 7,1 & 3,5 & 3,5 & 71,4 \\
\hline Suegra & 10,7 & 14,2 & 21,4 & 14,2 & 17,8 & 21,4 \\
\hline Suegro & 10,7 & 7,1 & 17,8 & 7,1 & 7,1 & 50 \\
\hline Pediatra & 0 & 3,5 & 7,1 & 35,7 & 50 & 3,5 \\
\hline Enfermera & 0 & 0 & 3,5 & 10,7 & 85,7 & 0 \\
\hline Comadrona & 0 & 7,1 & 7,1 & 25 & 21,4 & 37,9 \\
\hline Ginecóloga & 3,5 & 0 & 3,5 & 10,7 & 17,8 & 64,28 \\
\hline
\end{tabular}

Respecto al AS técnico formal, la totalidad de las mujeres consideraba que no procede en el caso de la ginecóloga (100\%), seguida de la comadrona $(92,8 \%)$ y del pediatra (100\%). En el caso de la enfermera este porcentaje llega al $71,4 \%$.

En el AS técnico informal, la mayoría de las mujeres se manifiestan muy satisfechas-completamente satisfechas cuando este apoyo procede de la pareja $(89,2 \%)$. En cambio, los porcentajes son menores en el caso de la madre $(32,1 \%)$ y del padre $(21,3 \%)$. (Tabla 3$)$.

Tabla 3: AS Técnico

\begin{tabular}{|l|c|c|c|c|c|c|}
\hline \multicolumn{1}{|c|}{ TÉCNICO } & $\begin{array}{c}\text { No } \\
\text { satisfecha }\end{array}$ & $\begin{array}{c}\text { Algo } \\
\text { satisfecha }\end{array}$ & $\begin{array}{c}\text { Moderadamente } \\
\text { satisfecha }\end{array}$ & $\begin{array}{c}\text { Muy } \\
\text { satisfecha }\end{array}$ & $\begin{array}{c}\text { Completamente } \\
\text { satisfecha }\end{array}$ & $\begin{array}{c}\text { No } \\
\text { procede }\end{array}$ \\
\hline Pareja & 0 & 0 & 7,1 & 10,7 & 78,5 & 3,5 \\
\hline Madre & 7,1 & 0 & 0 & 7,1 & 25 & 60,7 \\
\hline Padre & 3,5 & 3,5 & 3,5 & 3,5 & 17,8 & 67,8 \\
\hline Hermana & 3,5 & 3,5 & 3,5 & 3,5 & 14,2 & 71,4 \\
\hline Hermano & 0 & 3,5 & 7,1 & 3,5 & 0 & 85,7 \\
\hline Amigos & 7,1 & 0 & 3,5 & 3,5 & 10,7 & 75 \\
\hline Vecino & 0 & 0 & 3,5 & 3,5 & 0 & 92,8 \\
\hline Suegra & 3,5 & 0 & 3,5 & 7,1 & 10,7 & 75 \\
\hline Suegro & 3,5 & 3,5 & 7,1 & 10,7 & 0 & 75 \\
\hline Pediatra & 0 & 0 & 0 & 0 & 0 & 100 \\
\hline Enfermera & 0 & 0 & 0 & 28,5 & 0 & 71,4 \\
\hline Comadrona & 0 & 0 & 0 & 7,1 & 0 & 92,8 \\
\hline Ginecóloga & 0 & 0 & 0 & 0 & 0 & 100 \\
\hline
\end{tabular}

Respecto al AS emocional formal, la mayoría de las mujeres se manifiestan muy satisfechas-completamente satisfechas cuando este apoyo procede de la enfermera $(89,2 \%)$, pero decrece cuando procede del pediatra $(49,9 \%)$.

El AS emocional se considera que no procede, en la mayoría de los casos, cuando proviene de la ginecóloga $(89,2 \%)$ y de la comadrona $(84,1 \%)$ 
En el AS emocional informal, la mayoría de las mujeres se manifiestan muy satisfechas-completamente satisfechas cuando este apoyo proviene de la pareja $(78,4 \%)$, seguido de la madre $(60,4 \%)$, de los amigos y hermana $(35,6 \%)$ y del padre $(21,3 \%)$. (Tabla 4$)$

Tabla 4: AS Emocional

\begin{tabular}{|l|c|c|c|c|c|c|}
\hline EMOCIONAL & $\begin{array}{c}\text { No } \\
\text { satisfecha }\end{array}$ & $\begin{array}{c}\text { Algo } \\
\text { satisfecha }\end{array}$ & $\begin{array}{c}\text { Moderadamente } \\
\text { satisfecha }\end{array}$ & $\begin{array}{c}\text { Muy } \\
\text { satisfecha }\end{array}$ & $\begin{array}{c}\text { Completamente } \\
\text { satisfecha }\end{array}$ & $\begin{array}{c}\text { No } \\
\text { procede }\end{array}$ \\
\hline Pareja & 0 & 0 & 10,7 & 14,2 & 64,2 & 10,7 \\
\hline Madre & 3,5 & 0 & 14,2 & 7,1 & 53,3 & 21,4 \\
\hline Padre & 3,5 & 14,2 & 3,5 & 3,5 & 17,8 & 57,1 \\
\hline Hermana & 3,5 & 3,5 & 3,5 & 7,1 & 28,5 & 53,5 \\
\hline Hermano & 0 & 3,5 & 3,5 & 3,5 & 3,5 & 85,7 \\
\hline Amigos & 0 & 3,5 & 14,2 & 14,2 & 21,4 & 44,8 \\
\hline Vecino & 0 & 0 & 0 & 0 & 0 & 100 \\
\hline Suegra & 3,5 & 7,1 & 14,2 & 0 & 3,5 & 71,4 \\
\hline Suegro & 7,1 & 0 & 7,1 & 0 & 0 & 85,7 \\
\hline Pediatra & 3,5 & 7,1 & 0 & 21,4 & 28,5 & 37,9 \\
\hline Enfermera & 0 & 0 & 7,1 & 10,7 & 78,5 & 3,5 \\
\hline Comadrona & 0 & 3,5 & 3,5 & 3,5 & 7,1 & 84,1 \\
\hline Ginecóloga & 3,5 & 0 & 3,5 & 3,5 & 0 & 89,2 \\
\hline
\end{tabular}

Respecto al AS evaluativo formal, la mayoría de las mujeres se manifiestan muy satisfechas-completamente satisfechas cuando este apoyo procede de la enfermera $(89,2 \%)$, pero decrece en el caso del pediatra $(39,2 \%)$.

En el AS evaluativo informal, la mayoría de las mujeres se manifiestan muy satisfechas-completamente satisfechas cuando este apoyo proviene de la pareja $(93,1 \%)$, seguido de la madre $(62,7 \%)$, de los amigos $(53,5 \%)$ y de la hermana $(35,6 \%)$. Para más información consultar tabla 5.

Tabla 5: AS Evaluativo

\begin{tabular}{|l|c|c|c|c|c|c|}
\hline EVALUATIVO & $\begin{array}{c}\text { No } \\
\text { satisfecha }\end{array}$ & $\begin{array}{c}\text { Algo } \\
\text { satisfecha }\end{array}$ & $\begin{array}{c}\text { Moderadamen } \\
\text { te satisfecha }\end{array}$ & $\begin{array}{c}\text { Muy } \\
\text { satisfecha }\end{array}$ & $\begin{array}{c}\text { Completamente } \\
\text { satisfecha }\end{array}$ & $\begin{array}{c}\text { No } \\
\text { procede }\end{array}$ \\
\hline Pareja & 3,5 & 0 & 0 & 35,7 & 57,4 & 3,5 \\
\hline Madre & 7,1 & 0 & 7,1 & 21,4 & 41,3 & 21,4 \\
\hline Padre & 7,1 & 3,5 & 3,5 & 10,7 & 28,5 & 44,8 \\
\hline Hermana & 3,5 & 3,5 & 7,1 & 17,8 & 17,8 & 50 \\
\hline Hermano & 0 & 10,7 & 7,1 & 0 & 10,7 & 71,4 \\
\hline Amigos & 3,5 & 3,5 & 3,5 & 25 & 28,5 & 35,7 \\
\hline Vecino & 0 & 0 & 10,7 & 0 & 0 & 89,2 \\
\hline Suegra & 7,1 & 3,5 & 14,2 & 10,7 & 10,7 & 53,5 \\
\hline Suegro & 7,1 & 3,5 & 7,1 & 3,5 & 10,7 & 67,8 \\
\hline Pediatra & 0 & 0 & 17,8 & 32,1 & 7,1 & 41,3 \\
\hline Enfermera & 0 & 0 & 10,7 & 35,7 & 53,5 & 0 \\
\hline Comadrona & 0 & 3,5 & 3,5 & 10,7 & 7,1 & 75 \\
\hline Ginecóloga & 7,1 & 0 & 3,5 & 0 & 0 & 89,2 \\
\hline
\end{tabular}


Por último se abordan los datos cualitativos, que son los que provienen de los motivos por el que los proveedores de AS no satisfacían completamente las necesidades de las entrevistadas.

Las categorías se agruparon según los proveedores del AS.

Para el AS informal se identificaron 8 categorías: disparidad y/o actualización en los consejos, exigencia, empatía, confianza, inseguridades, tiempo, distancia y no procede:

Disparidad y/o actualización en los consejos.

"Con mis amigos estoy moderadamente satisfecha porque hay opiniones diferentes: algunas me convencen más que otras". "Con mi madre estoy moderadamente satisfecha porque las cosas ya han cambiado y ella te dice que haga las cosas de una manera y en cambio los médicos te piden que las hagas de otro modo". "Los amigos que no tienen hijos te dan consejos que no son muy reales". "Mi cuñada: porque es la que ha tenido un bebé más reciente y le hago preguntas más concretas. Esta información que me da me hace sentirme bien, pero es moderadamente satisfecha porque los niños son diferentes y lo que le ha servido a ella a mí no".

\section{Exigencia.}

"Mi madre es más dura incluso para decir las cosas y si algo no lo ve bien te lo dice y a veces no quiero que las cosas me las diga así. "El refuerzo que he recibido de mi madre es moderado porque ella es más exigente". "No siempre te dicen la palabra refuerzo. A veces lo intuyes y te gustaría que te lo dijeran".

\section{Empatía.}

"Es más la percepción mía, sobre todo la pareja lo que tú vives no es lo que él vive. El que uno se ponga en el lugar del otro, sobre todo al principio es muy difícil por eso es moderadamente satisfecha". "Hay cosas que a veces mi marido no entiende, en cambio mi hermana sí al tener los hijos"

\section{Confianza.}

"El nivel de confianza con la suegra no es el mismo que con la madre". "A mi marido hay cosas que no se las puedo comentar. Algún tema con su madre o con su hermano, temas familiares que son más complicados y entonces busco a mi madre. Pero no todo se lo puedo contar a mi madre y tampoco quiero que coja manía a mi marido y entonces se lo cuento a mi amiga, por eso estoy bastante satisfecha con todos". 
Inseguridades.

"Mi marido, hay veces que pienso que podría ayudar un poco más. Cuando la niña llora es mi niña. Él se pone más nervioso que yo, pero es consciente, él lo reconoce, cuando llora me dice "es que no puedo", pero en el baño, ahí sí que me ayuda".

Tiempo.

"Estoy moderadamente satisfecha con la ayuda que recibo de mi pareja porque tiene el hándicap del tiempo. Es cierto que cuando está en casa está con el niño, pero si no es por el tiempo". "Mis padres trabajan mucho y no puedo tener de ellos todo el soporte que me gustaría". "Los tíos vienen en un momento dado pero es puntual".

Distancia.

"Tengo a toda la familia fuera. Me comunico con ellos por teléfono o por internet".

No procede.

"Mi pareja me ayuda, pero no me aconseja por eso no procede" "Mi padre me ayuda pero no me aconseja" "Mi padre no procede porque falleció" "Mi madre no se mete".

Para el AS formal se identificaron 4 categorías: contacto profesional/accesibilidad, disparidad y/o actualización, confianza y no procede:

Contacto profesional- Accesibilidad.

"Con el pediatra he tenido menos visitas de las que me gustaría, a diferencia de con la enfermera que la satisfacción va unida al número de visitas de seguimiento". "El parto no lo llevé por la seguridad social y apenas tuve contacto con la comadrona". "Al pediatra lo he visto poco, pero cuando lo he visto es muy satisfactorio". "La ginecóloga y la comadrona no estoy muy contenta, tengo que coger el tren o el autobús porque tengo que ir a ver a mi comadrona que está lejos. Este desplazamiento me va mal y la distancia física me corta". "He tenido poco contacto después del parto (refiriéndose a ginecóloga $y$ comadrona). Para mi a más contacto, más satisfacción"

Disparidad y/o actualización de consejos.

"La enfermera no puede contestar a todas las dudas que tengo". "La información muy bien pero a veces una persona te dice blanco y otra te dice negro".

Confianza.

"Conforme pasaba el tiempo me he abierto más con la enfermera, ha habido más intimidad". "Yo con ningún sanitario he establecido este 
tipo de relación en la que ofrezca estos sentimientos". "No puedo tener con la enfermera el nivel de intimidad que con las personas más cercanas".

No procede.

"La ginecóloga no procede, porque se centraba sobre todo en el embarazo y después no ha habido seguimiento". "Con la comadrona y ginecóloga desde que nació la niña no tengo relación, por lo que no tengo ese refuerzo" "La comadrona y la ginecóloga fui por lo privado y fue muy bien. Era muy amiga de mi madre y era conocida y claro me explicaba todo, pero se centraba sobre todo en el embarazo. Una vez tienes a la niña pasas a otra etapa"

\section{DISCUSIÓN}

A continuación, se mencionarán los resultados más relevantes y se compararán con investigaciones previas; aportando nuevos datos que resultan de interés.

La muestra curiosamente ha resultado ser homogénea en cuanto a nacionalidad, edad de las madres, estado civil y estudios. Estos factores pueden producir diferencias en los resultados y sesgar las conclusiones dado que, por ejemplo, en relación a la nacionalidad se han observado mayores tasas de embarazos adolescentes, prematuridad y bajo peso ${ }^{(14)}$, además de un menor apoyo social y mayores trastornos psicológicos ${ }^{(15)}$ en mujeres inmigrantes.

Si mencionamos la presencia/ausencia de pareja, Mercer indica que la presencia del padre o compañero "contribuye al proceso de adopción del rol maternal como no puede hacerlo ninguna otra persona" ${ }^{(4)}$. Además, Beck identifica el estado marital y su naturaleza como factores de riesgo (tamaño efecto: pequeño) para la depresión postparto. Con el mismo tamaño de efecto, también señala la situación socioeconómica (ingresos, educación y ocupación) ${ }^{(4)}$.

Por criterios de edad, estas madres están alejadas de la maternidad adolescente; etapa que ha sido estudiada con interés al ser considerada un problema social y sanitario ${ }^{(16,17)}$.

Seguidamente, se comentarán los hallazgos relacionados con las características del apoyo; teniendo en cuenta que la muestra está limitada por la saturación de datos a nivel cualitativo. Se sugieren nuevas investigaciones, con metodología cuantitativa y con un muestreo más elevado y multicéntrico que no comprometa la validez externa del estudio.

En líneas generales, y centrándonos en primer lugar en el AS formal, se observa, tal y como indican otros estudios al identificar a las enfermeras entre las redes de apoyo ${ }^{(8)}$ importantes ${ }^{(4)}$ y evaluadas con un nivel de satisfacción buena 0 excelente ${ }^{(18)}$, que las madres están muy satisfechas-completamente satisfechas con el AS ofrecido por éstas, sobre todo a nivel informativo y emocional, todo y que alguna de ellas manifestaba no querer compartir emociones correspondientes a partes más íntimas de su vida con personas que no sean de su entorno más próximo. 
Dado que la conducción de grupos la realizaba una enfermera, sola o en colaboración con otros profesionales, puede deducirse por las aportaciones de las participantes, que esto ha repercutido en la evaluación. El tiempo que ha dedicado este profesional a las madres y la mayor accesibilidad y contacto a través del grupo ha favorecido su satisfacción, y ha contribuido favorablemente a una mayor confianza percibida, creciendo conforme se realizaban más contactos. Esta percepción coincide con otros estudios, en el que el factor "tiempo" debía considerarse en la mejora de la comunicación entre primíparas y personal sanitario $^{(19)}$.

En cambio, el grado de satisfacción valorado en profesionales como la comadrona y ginecóloga es mucho menor, siendo la accesibilidad y el contacto profesional categorías que influyen en esta valoración. En este sentido podemos identificar un sesgo de memoria, pues a más edad tenga el bebé, menos contacto se producirá con estos profesionales que cuando las madres estaban embarazadas y más percepción de alejamiento en el contacto profesional tendrán. No obstante, el "poco contacto con el profesional" ha sido identificado por algunas madres como un "no procede". Estos resultados son contrarios, pero congruentes con el sesgo de memoria mencionado, si los relacionamos con investigaciones previas, en el que el mejor profesional valorado era la comadrona, debido al momento de la maternidad en que se realizaba el estudio: 4-6 semanas desde la fecha del parto $^{(19)}$.

La poca satisfacción con AS formal informativo, coincidente con otros estudios ${ }^{(19)}$, también se produce ante una disparidad y/o falta de actualización de consejos, lo que provoca desconfianza y recelo de las madres hacia los profesionales.

Si proseguimos con los resultados del AS evaluativo e instrumental en el primero, las madres consideran que cuando los profesionales les informan de un crecimiento y desarrollo correcto de su hijo, indirectamente significa que ellas lo están haciendo bien, relacionando esta percepción satisfactoria con AS evaluativo. En cuanto al segundo, los profesionales tienen poco que aportar. Las madres han identificado este AS en la ayuda que han recibido por los profesionales en tareas en el trascurso del taller: preparar biberones, acunar al niño mientras se mostraba intranquilo, entre otras cosas.

Profundizando en la red de AS informal, es decir, en familiares, amigos y vecinos, se observa que la pareja y las madres (abuela materna) son las mejores evaluadas, tal y como sugiere la investigación previa ${ }^{(4,13,20,21)}$, en todos los tipos de apoyo ya sea informativo, técnico, emocional y evaluativo. Las situaciones que han llevado a valoraciones más bajas en estos casos se debe, al igual que en el AS formal, a disparidad y/o actualización y/o confianza en los consejos. Además se añade la falta de tiempo por motivos laborales que limitan la disponibilidad del AS, el nivel de exigencia, el grado de confianza originado por la sensación de estar "en deuda" al percibir que la ayuda recibida se trata de un favor que debe retornarse y la falta de empatía.

En el caso del padre, hermanas y amigos las valoraciones han sido muy similares, pero menores que en la pareja y la madre (abuela materna). Al respecto, destacar que las figuras maternas siempre han sido importantes y mejor evaluadas, al igual que en la investigación previa ${ }^{(22,23)}$; puntualizando las entrevistadas, que se trataba de amigas y no de amigos e introduciendo, en algún caso, la figura de la 
cuñada. Esto puede deberse a los comentarios referidos a la sensación de empatía que sentían de estas mujeres que ya habían tenido experiencia en la maternidad; todo y que lo que les valía a unas a otras no.

Los suegros, suegra y vecinos suelen tener puntuaciones más bajas con respecto al resto. La percepción de la satisfacción del suegro y de los vecinos "no procede" en casi la totalidad de los casos, encontrando entre los motivos la enfermedad, defunción, lejanía o pérdida de contacto, entre otros.

Finalmente, las madres que participan en el grupo aseguran tener más apoyo en todos los sentidos "Siempre encontré a faltar el estar al día de los cursos que se hacen aquí. Al principio estaba sola y era novata, a quienes se inicia en esto necesita apoyo... si participas más del grupo, la experiencia de otras madres te ayudan a resolver dudas". Estos resultados coinciden con los aportados por

Wilkins ${ }^{(24)}$, que observa entre los beneficios del participar en el grupo: el compartir experiencias, pedir ayuda sin miedo a la crítica, normalizando la transición a la maternidad y facilitando el aprendizaje hacia una nueva experiencia.

Sería interesante abrir nuevas líneas de investigación que compararan la satisfacción de las madres que participen en dinámicas grupales versus a las que sólo acuden a las consultas establecidas en programas de promoción de la salud y prevención de la enfermedad. También sería interesante valorar, antes de la participación en estas dinámicas grupales, el perfil del AS de las madres integrantes del grupo, con el objetivo de comparar la percepción de la satisfacción del AS recibido antes y después de su participación; y si el grupo le ha ayudado a percibir mejor los AS independientemente de su procedencia.

\section{CONCLUSIONES}

En relación al AS formal, la mayoría de las madres están muy satisfechascompletamente satisfechas con el AS informativo, emocional y evaluativo procedente de las enfermeras; contribuyendo en esta percepción aspectos como la disparidad y/o actualización de consejos, la accesibilidad y contacto profesional, así como el grado de confianza con el profesional.

En relación al AS formal, la mayoría de las madres están muy satisfechascompletamente satisfechas con el AS informativo, emocional, evaluativo y técnico procedente de la pareja y de la madre (abuela materna); contribuyendo en esta percepción aspectos como disparidad y/o actualización de consejos, exigencia, empatía, confianza, inseguridades, tiempo y distancia.

Todos estos factores deberían ser tenidos en cuenta en los planes de mejora de la satisfacción y acompañamiento de las madres en este momento de profundos cambios en todos los aspectos de su vida, dado que aportar un AS satisfactorio repercute en la promoción de la salud y en la prevención de la enfermedad.

\section{BIBLIOGRAFÍA}

1-Canabal GE, Jaramillo CD, Rosero DH, Valencia MG. La teoría de las transiciones y la salud de la mujer en el embarazo y en el postparto. Aquichan.2007; 7 (1): 8-23 
2- Berlanga S, Pérez RM, Vizcaya MF. Aplicación práctica de los modelos de Mercer y Beck en las especialidades de enfermería- Evidentia. 2012. 9 (39). [Consultado 5 de enero 2013]; Disponible en: http://www.indexf.com/evidentia/n39/ev7722.php.

3- Marrimer A, Raile M. Modelos y teorías en enfermería. 7nd.ed. Madrid: Elsevier; 2011.

4- Leahy P. Social Support for first-time mothers. An Irish study. MCN AM J Matern Child Nurs. 2007; 32 (6): 368-74.

5- Ayala A, Rodriguez C, Frades B, Forjaz MJ, Martínez P, Fernández G, Rojo F. Propiedad psicométricas del Cuestionario de Apoyo Social Funional y de la Escada de Soledad en adultos mayores no institucionalizados en España. Gac Sanitaria. 2012; 26 (4): 317-324

6- Leahy P, McCarthy G, Corcoran P. First-time mothers: social support, maternal parental self-efficacy and postnatal depression. Journal of Clinical Nursing. 2011; 21: 388-397

7- Terol MC, López S, Neipp MC, Rodríguez J, Pastor MA, Martín-Aragón M. Apoyo social e instrumentos de evaluación: revisión y clasificación. Anuario de Psicología. 2004; 35 (1): 23-45.

8- López I, Menéndez S, Lorence B, Jiménez L, Hidalgo MV, Sánchez J. Evaluación del apoyo social mediante la escala ASSIS: descripción y resultados en una muestra de madres en situación de riesgo psicosocial. Intervención psicosocial. 2007; 16 (3): 323-337.

9- Moreno MG, Interial MG, Sauceda PF, Vázquez L, López JT. Satisfacción de las madres con la atención a sus hijos hospitalizados. Aquichan. 2011; 11(1): 4047.

10- Moreno ME. ¿Qué esperan las personas del cuidado de enfermería? Aquichan. 2010; 10 (2): 96-98.

11- Sánchez F, Sánchez FJ, López Y. Un estudio cualitativo para conocer las características que definen y mejoran la formación en comunicación asistencial e interprofesional. Rev Calidad Asistencial. 2008; 23 8(5): 199-204.

12- Campos P. Barbosa MA, fernandes G. The study of the phenomenology as one way of access for the improvement of the nursing assistance Cultura de los Cuidados. 2011. 29: 9-15

13- Burnard P. A method of analysing interview transcripts in qualitative research. Nurse Education Today. 1991; 11, 461-466.

14-Río Isabel, Castelló Adela, Jané Mireia, Prats Ramón, Barona Carmen, Más Rosa et al . Indicadores de salud reproductiva y perinatal en mujeres inmigrantes y autóctonas residentes en Cataluña y en la Comunitat Valenciana (2005-2006). Gac Sanit [revista en la Internet]. 2010 Abr [Consultado 5 de enero 2013] ; 24(2): 123-127. Disponible en: http://scielo.isciii.es/scielo.php?script=sci_arttext\&pid=S0213-

91112010000200007\&lng=es.

15- Pérez F. Las mujeres inmigrantes y su respuesta psicológica al puerperio inmediato. Diferencias obstétricas y sociodemográficas [tesis doctoral] . Universidad de Granada. Separtamento de enfermería. 2011. [Consultado el 5 de enero 2013]. Disponible en: http://hera.ugr.es/tesisugr/20761181.pdf.

16- Calesso M. Vínculo afectivo y estrés en la maternidad adolescente: un estudio con metodología combinada [tesis doctoral]. Universidad Autónoma de Barcelona. Facultad de Piscología,. Departamento de psicología clínica y de la salud. 2007. [Consultado el 5 de enero 2013]. Disponible en: http://www.tdx.cat/bitstream/handle/10803/5459/mcm1de1 .pdf?sequence=1 
17.-Pascual V, Toirac AS. Patrón de riesgo en la gestante adolescente primípara: un problema latente. MEDISAN [revista en la Internet]. 2011;15(12): 1720-1728 [consultado 2 de enero 2013]. Disponible en: http://scielo.sld.cu/scielo.php?script=sci_arttext\&pid=S102930192011001200006\&lng=es.

18- Moreno R, Villarreal E, Hernández MA, Gallegos R. Percepción de la satisfacción del usuario con la atención del niño por la enfermera materno.-infantil. Desarrollo Cientif Enferm. 2009; 17 (9): 400-403

19- Díaz J, Catalán D, Fernández MM, Granados G. La comunicación y la satisfacción de las primíparas en un servicio público de salud. Gac. Sanit. 2011; 25(6): 483-489.

20- Cing Ch, Shu K, Yu C, Hsiu Ch. Postpartum Taiwanese women: their postpartum depression, social support and health-promoting lifestyle profiles. Midwifery. 2007; 16 (8): 1550-60

21-Leahy P. First-time mothers: social support and confidence in infant care. Journal of advanced Nunrsing. 2005; 50 (5): 479-488.

22- Goméz SL. Representaciones maternales en madres primerizas que presentan sintomatología depresiva postparto [tesis doctoral]. Universidad Católica de Perú. Facultad de letras y ciencias humanas. 2011. [Consultado 5 de enero 2013]. Disponible en: http://tesis.pucp.edu.pe/repositorio/handle/123456789/1197

23- Stern D. El nacimiento de una madre: cómo la experiencia de la maternidad te hará cambiar para siempre. Barcelona: Editorial Paidós; 1999.

24- Wilkins A. A qualitative study exploring the dupport needs of first-time mothers on their journey towards intuitive parenting. Midwifery.2006; 22 (2): 169-80. 